\title{
Pathway- and clinical-factor-based risk model predicts the prognosis of patients with gastric cancer
}

\author{
JUNCHI YANG $^{1 *}$, LUMIN BO $^{2 *}$, TING HAN $^{1 *}$, DAN DING $^{1}$, MINGMING NIE $^{1}$ and KAI YIN ${ }^{1}$ \\ Departments of ${ }^{1}$ Gastrointestinal Surgery and ${ }^{2}$ Gastroenterology, Changhai Hospital, Shanghai 200433, P.R. China
}

Received September 30, 2017; Accepted January 25, 2018

DOI: $10.3892 / \mathrm{mmr} .2018 .8722$

\begin{abstract}
Gastric cancer (GC) has a high incidence and mortality rate. If discovered late, GC tends to have a poor prognosis. Improvements in the prognostic accuracy of GC through combined analysis of multiple relevant genes and clinical factors may solve this problem. In the present study, GSE62254 (including 300 GC tissues), obtained from the Gene Expression Omnibus database, was used as a training set, and the mRNA-sequencing data of GC (including 384 GC tissues) downloaded from the Cancer Genome Atlas database served as a validation set. Based on the t-test and Wilcoxon test, the significantly differentially expressed genes (DEGs) were obtained by screening the intersecting DEGs. The prognosis-associated genes and clinical factors were identified using Cox regression analysis in the $\mathrm{R}$ survival package. The optimal prognosis-associated pathways were examined using the Cox-proportional hazards (Cox-PH) model in the $\mathrm{R}$ penalized package. Finally, risk prediction models were constructed and validated using the Cox- $\mathrm{PH}$ model and the Kaplan-Meier method, respectively. There were a total of 382 significant DEGs, including 268 upregulated genes and 114 downregulated genes. A total of 50 prognosis-associated genes were identified, 16 optimal prognosis-associated pathways (including mitochondrial pathway and the tyrosine-protein kinase JAK-signal transducer and activator of transcription signaling pathway, which involve caspase 7, phosphoinositide-3-kinase regulatory subunit 3, peroxisome proliferator-activated receptor $\gamma$ and collagen triple helix repeat containing 1) and four prognosis-associated clinical factors [including Pathologic_N, Pathologic_stage, mutL
\end{abstract}

Correspondence to: Dr Mingming Nie or Dr Kai Yin, Department of Gastrointestinal Surgery, Changhai Hospital, 168 Changhai Road, Yangpu, Shanghai 200433, P.R. China

E-mail: niemm888@163.com

E-mail:kyin67@126.com

*Contributed equally

Key words: gastric cancer, differentially expressed genes, bidirectional hierarchical clustering, survival curve, risk prediction model homolog 1 (MLH1) mutation and recurrence]. The pathwayand clinical-factor-based risk prediction model exhibited marked prognostic accuracy. The clinical-factor-based risk prediction model with improved P-values for prognosis prediction may be superior to the pathway-based risk prediction model in predicting the prognosis of GC patients.

\section{Introduction}

Gastric cancer (GC, additionally termed stomach cancer) is derived from gastric mucosa (1), $60 \%$ of which is induced by infection with the bacterium Helicobacter pylori (2-4). GC is characterized by several early signs (including heartburn, lack of appetite, nausea and upper abdominal pain) and certain later symptoms (including weight loss, dysphagia, vomiting and yellowing of the whites of the eyes and skin) (5). If left untreated, GC may undergo diffusion transfer to other parts of the body, including the lungs, liver, lymph nodes and bones (6). The 5-year survival rate of patients with GC is $<10 \%$ worldwide, and late discovery of illness may the worsen prognosis (7). Globally, there were 950,000 new cases of GC and 723,000 mortalities in 2012 (3). Therefore, early diagnosis, reasonable prognostic assessment, and timely and appropriate intervention are very important to improving the outcomes of GC.

The study of large numbers of prognostic markers may guide the clinical monitoring of patients at a high risk of relapse, and further treatment may be administered to improve the survival rate of patients with GC. Previous studies have demonstrated that astrocyte elevated gene 1 overexpression serves as a promising prognostic factor for $\mathrm{GC}$, and targeted inhibition thereof may be a novel therapeutic strategy for the disease $(8,9)$. The expression of human epidermal growth factor receptor 2 can be used to predict sensitivity to trastuzumab-based chemotherapy and the overall survival of patients with advanced GC (10). Adenine-thymine-rich interactive domain $1 \mathrm{~A}$ is reported to be a potential prognostic marker and therapeutic target for GC $(11,12)$. The accuracies of these different biomarkers were not the same, thus more relevant prognostic factors are required. Okugawa et al (13) reported that the brain-derived neutrophic factor (BDNF)/neurotrophic receptor tyrosine kinase 2 (TrkB) axis has an association with the prognosis of patients with GC, and the BDNF/TrkB pathway may serve an important role in the progression of GC. At present, the prognosis of GC primarily depends on 
such factors as serum markers and the clinical condition of a patient $(14,15)$. Combining multiple relevant genes and clinical factors may improve prognostic accuracy in GC.

Huang et al (16) developed a novel computational model for breast cancer prognosis by combining the pathway deregulation score (PDS)-based pathifier algorithm, Cox proportional hazards regression and the L1-lasso penalization method to select promising targets for therapeutic intervention. Huang et al (17) developed a novel computational method that uses personalized PDS with pathway-based metabolomics data analysis for breast cancer diagnosis. However, few studies have reported the value of pathway and clinical factor-based risk models for GC prognosis. The present study adopted similar methods, and aimed to investigate the prognostic ability of different risk prediction models based on the identified pathways and clinical factors associated with the prognosis of GC (Fig. 1).

\section{Materials and methods}

Data source. Gene expression profiles of the GSE62254 dataset (18) [platform: GPL570 (HG-U133_Plus_2) Affymetrix (Thermo Fisher Scientific, Inc., Waltham, MA, USA) Human Genome U133 Plus 2.0 Array] were obtained from the Gene Expression Omnibus (GEO; www.ncbi.nlm. nih.gov/geo) database. GSE62254 consisted of 300 GC tissue samples and served as a training set in the present study. These GC tissue samples were obtained from patients during gastrectomy procedures at Samsung Medical Centre, Seoul, Korea (2004-2007). Another mRNA-sequencing dataset for GC [platform: Illumina, Inc. (San Diego, CA, USA) HiSeq 2000 RNA Sequencing] was downloaded from The Cancer Genome Atlas (TCGA; cancergenome.nih.gov). This mRNA-sequencing dataset, containing 384 GC tissue samples, was taken as a validation set. The demographic and clinical characteristics of all samples in the training and validation set are presented in Table I. In order to eliminate the technology bias in systematic measurement between the distinct datasets and platforms, these two datasets were independently standardized (19).

Data preprocessing and differentially expressed gene (DEG) screening. The background correction and data normalization of GSE62254 were performed using the oligo package (www .bioconductor.org/packages/release/bioc/html/oligo.html) (20) in $\mathrm{R}$ (21). Based on the prognostic information, samples were divided into two groups: A poor prognosis group (samples from patients who survived for $<12$ months and were deceased), and a good prognosis group (samples from patients who survived for $>60$ months and were alive). Subsequently, the t-test (127.0.0.1:26738/library/stats/html/t.test.html) (22) and the Wilcoxon test (127.0.0.1:26738/library/stats/html/wilcox.test .html) (23) in R were used for screening the genes that were significantly differentially expressed between the poor prognosis group and the good prognosis group. A false discovery rate $<0.05$ and llog fold changel $>0.263$ were considered to be the thresholds. Overlapping DEGs predicted by the t-test and Wilcoxon test were selected for further analysis.

Identification of prognosis-associated genes and clinical factors. Prognosis-associated genes and clinical factors were selected using univariate and multivariate Cox regression analysis in the $\mathrm{R}$ survival package (bioconductor.org/packages/survivalr) (24). A P-value $<0.05$ was set as the cut-off criterion. The expression values of the prognosis-associated genes were extracted to perform bidirectional hierarchical clustering (25) using the $\mathrm{R}$ heatmap package (cran.r-project .org/web/packages/pheatmap/index.html) (26). The purpose of the hierarchical clustering analysis was to intuitively observe the differences in prognosis-associated gene expression between samples.

Selection of prognosis-associated pathways. The Gene Set Enrichment Analysis (GSEA) database (www.broadinstitute .org/gsea) (27) is a microarray data analysis tool containing multiple functions and pathways. All the pathway annotation files of 217 Biocarta pathways and 186 Kyoto Encyclopedia of Genes and Genomes (KEGG) pathways in the GSEA database (27) were downloaded and taken as reference pathways. Subsequently, the expression matrix of the prognosisassociated genes was converted into a pathway deregulation score (PDS) matrix of the relevant pathways using the principal components analysis algorithm in the $\mathrm{R}$ pathifier package (bioconductor.org/packages/pathifier) (28). The optimal prognosis-associated pathways were screened subsequent to importing the PDS matrix using the Cox proportional hazards (Cox-PH) model in the $\mathrm{R}$ penalized package (bioconductor .org/packages/penalized) (29). The parameter 'lambda' was obtained upon performing 1,000 rounds of cross-validation likelihood (cvl) (30) circular calculation.

Construction and validation of risk prediction models. Based on the Cox-PH prognosis coefficients of the optimal prognosis-associated pathways, the pathway-based risk prediction model was constructed and the prognosis index (PI) score of each sample was calculated. According to the median of the PI scores, the samples in the training set were divided into high- and low-risk groups. The correlations between the risk prediction model and prognosis were estimated using the Kaplan-Meier (KM) method in the R survival package (24). In addition, the risk prediction model was validated using the validation set.

Using the Cox-PH model in the R penalized package (29), the optimal prognosis-associated genes were identified following importing of the gene expression matrix of the prognosis-associated genes. The gene-based risk prediction model was built and the PI score of each sample was calculated based on the Cox-PH prognosis coefficients of the optimal prognosis-associated genes. The median of the PI scores was considered the demarcation point, and the samples in the training set were additionally divided into high- and low-risk groups. Using KM survival curves, the correlations between the gene-based risk prediction model and prognosis were evaluated in the training set and the validation set. The predictive effects of the gene-based risk prediction model were compared to those of the pathway-based risk prediction model.

Using the Cox-PH model, the prognosis coefficients of the prognosis-associated clinical factors were determined, and a clinical factor-based risk prediction model was constructed. The PI scores of the samples were calculated. Subsequently, the samples in the training set were divided 


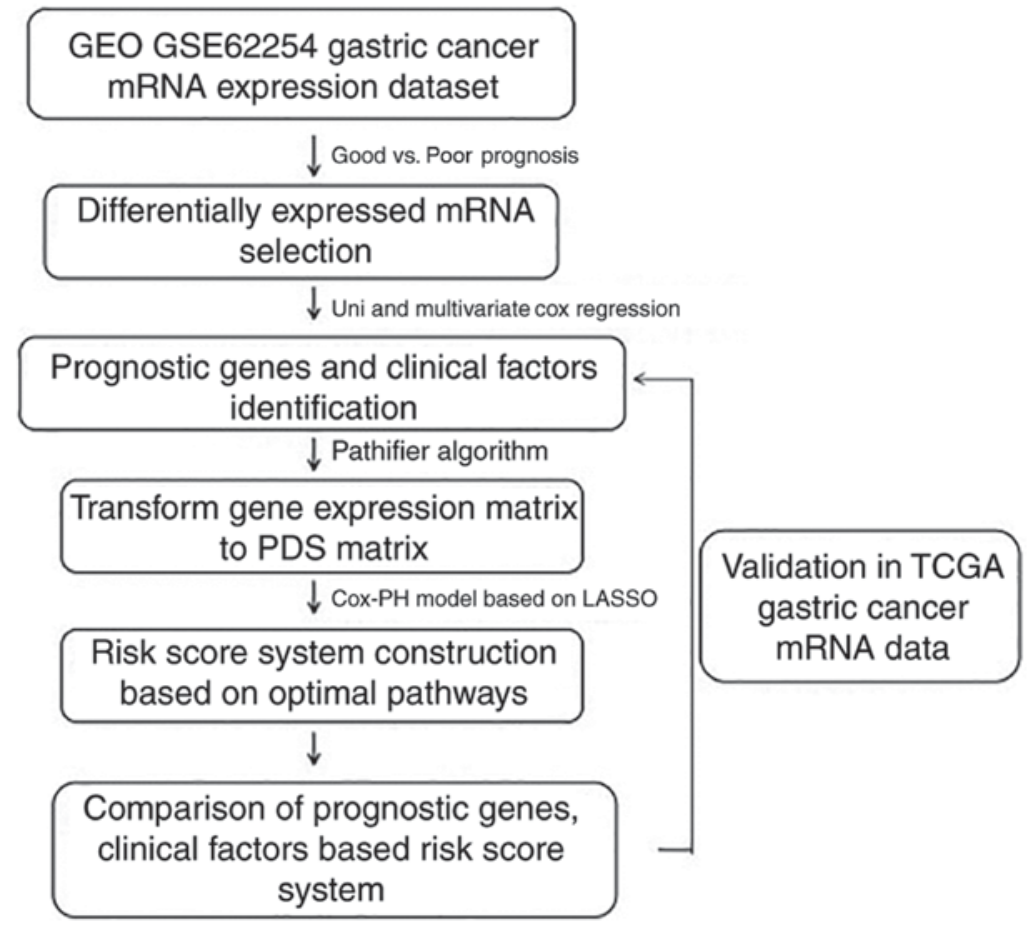

Figure 1. Workflow diagram indicating the process included in the analysis. GEO, Gene Expression Omnibus; TCGA, The Cancer Genome Atlas; PDS, pathway deregulation score; Cox-PH, Cox-proportional hazards.

Table I. Clinical characteristics of patients in the training set (GSE62254) and the validation set (TCGA dataset).

\begin{tabular}{lcc}
\hline Clinical characteristics & GSE62254 $(\mathrm{n}=300)$ & TCGA (n=384) \\
\hline Age, years (mean \pm standard deviation) & $61.94 \pm 11.36$ & $65.15 \pm 10.61$ \\
Sex, male/female & $199 / 101$ & $243 / 133 / 8$ \\
Pathologic_M, M0/M1/- & $273 / 27$ & $341 / 19 / 24$ \\
Pathologic_N, N0/N1/N2/N3 & $38 / 131 / 80 / 51$ & $118 / 100 / 77 / 16$ \\
Pathologic_T, T1/T2/T3/T4/- & $2 / 186 / 91 / 21$ & $20 / 74 / 172 / 107 / 11$ \\
Pathologic_stage, I/II/III/IV/- & $30 / 96 / 95 / 77 / 2$ & $51 / 116 / 174 / 31 / 12$ \\
Pathology type, diffuse/intestinal/mixed/- & $134 / 146 / 17 / 3$ & - \\
MLH1 mutation, yes/no/- & $234 / 64 / 2$ & $18 / 366$ \\
EBV infection, yes/no/- & $18 / 257 / 25$ & - \\
Recurrence, yes/no/- & $125 / 157 / 18$ & $78 / 260 / 46$ \\
Venous invasion, yes/no/- & $44 / 129 / 127$ & - \\
Lymphatic lymphovascular invasion, yes/no/- & $205 / 73 / 22$ & - \\
Subtypes, MSS-TP53/MSS-TP53+MSI-EMT/- & $107 / 79 / 68 / 46$ & - \\
Mortality, deceased/alive/- & $135 / 148 / / 17$ & $122 / 238 / 24$ \\
Disease-free survival, months (mean \pm standard deviation) & $33.72 \pm 29.82$ & $15.84 \pm 17.05$ \\
Overall survival time, months (mean \pm standard deviation) & $50.59 \pm 31.42$ & $16.17 \pm 16.96$ \\
\hline
\end{tabular}

TCGA, The Cancer Genome Atlas; MLH1, mutL homolog 1; EBV, Epstein-Barr virus; MSS-TP53-, Microsatellite stable-tumor protein 53-inactive; MSS-TP53 ${ }^{+}$, microsatellite stable-tumor protein 53-active; MSI-EMT, microsatellite instable-epithelial to mesenchymal transition.

into high- and low-risk groups, with their median PI as the demarcation point. Using KM survival curves, the correlations between the clinical-factor-based risk prediction model and prognosis were assessed in the training set and the validation set. Furthermore, the predictive effects of the clinical factor-based risk prediction model were compared with those of the pathway-based risk prediction model.

When the Cox-PH prognosis coefficients of the optimal prognosis-associated pathways had been integrated with those of the prognosis-associated clinical factors, a risk prediction 
model was constructed based on clinical factors and pathways. The PI score of each sample was calculated, and their PI median was taken as the demarcation point to divide the samples in the training set into high- and low-risk groups. Additionally, the correlations between the risk prediction model and prognosis were estimated in the training set and the validation set. Finally, the predictive effects of the risk prediction model based on clinical factors and pathways were compared with those of the pathway-based risk prediction model.

\section{Results}

$D E G$ screening. According to the prognostic information, 48 samples were classified into a poor prognosis group and 58 samples into a good prognosis group. A total of 617 DEGs and 671 DEGs were identified by the t-test and Wilcoxon test, respectively. The 382 overlapping DEGs (268 upregulated genes and 114 downregulated genes) were used for further analysis.

Identification of the prognosis-associated genes and clinical factors. A total of 50 prognosis-associated genes (Table II) and four prognosis-associated clinical factors [including Pathologic_N, Pathologic_stage, mutL homolog 1 (MLH1) mutation, and recurrence] (Table III) were screened based on the Cox regression analysis. Samples were divided into group 1 (including $173 \mathrm{GC}$ samples) and group 2 (including $127 \mathrm{GC}$ samples) according to the clustering analysis of prognosis-associated genes (Fig. 2). Moreover, significant differences were observed in the recurrence $\left(\mathrm{P}=1.29 \times 10^{-7}\right)$, Pathologic_N $\left(\mathrm{P}=1.77 \times 10^{-2}\right)$, Pathologic_stage $\left(\mathrm{P}=1.52 \times 10^{-3}\right)$, and MLH1 mutation $\left(\mathrm{P}=4.61 \times 10^{-2}\right)$ between the two groups. Group 1 had less recurrence, less lymphatic metastasis, lower tumor stage and more MLH1 mutations compared with group 2 .

Selection of prognosis-associated pathways. The expression matrix of the 50 prognosis-associated genes was converted into a PDS matrix, and 118 GC-associated pathways (including 26 Biocarta pathways and 92 KEGG pathways) were selected. Based on cvl circular calculation, the maximum value of cvl was -965.3297 (parameter 'lambda'=0.9361) (Fig. 3). Furthermore, 16 optimal prognosis-associated pathways including four Biocarta pathways (including the mitochondrial pathway) and 12 KEGG pathways [including the tyrosine-protein kinase JAK (JAK)-signal transducer and activator of transcription (STAT) signaling pathway] were obtained using the Cox-PH model with this parameter value (Table III). Meanwhile, 10 prognosis-associated genes [caspase 7 (CASP7), myosin heavy chain 14 (MYH14), nicotinamide nucleotide adenylyltransferase 1 (NMNAT1), phosphoinositide-3-kinase regulatory subunit 3 (PIK3R3), peroxisome proliferator activated receptor $\gamma$ (PPARG), tight junction protein 3 (TJP3), cation channel sperm associated auxiliary subunit $\beta$ (CATSPERB), CDC43 effector protein 5 (CDC42EP5); collagen triple helix repeat containing 1 (CTHRC1), and dehydrogenase/reductase 11 (DHRS11)] were involved in these 16 optimal prognosis-associated pathways.

The samples in GSE62254 were divided into group I (including 166 GC samples) and group II (including
Table II. Prognosis-associated genes $(n=50)$ identified by the Cox regression analysis.

\begin{tabular}{|c|c|c|}
\hline Gene & $\begin{array}{c}\text { Univariate cox } \\
\text { P-value }\end{array}$ & $\begin{array}{c}\text { Multivariate cox } \\
\text { P-value }\end{array}$ \\
\hline TTC38 & $4.70 \times 10^{-02}$ & $3.62 \times 10^{-06}$ \\
\hline DNAJC16 & $1.30 \times 10^{-04}$ & $1.87 \times 10^{-05}$ \\
\hline RAB11FIP4 & $3.40 \times 10^{-06}$ & $1.09 \times 10^{-04}$ \\
\hline CDC42EP5 & $1.00 \times 10^{-08}$ & $5.42 \times 10^{-04}$ \\
\hline MYH14 & $3.20 \times 10^{-06}$ & $6.85 \times 10^{-04}$ \\
\hline LRRC31 & $3.00 \times 10^{-02}$ & $7.77 \times 10^{-04}$ \\
\hline SIAE & $2.90 \times 10^{-06}$ & $1.53 \times 10^{-03}$ \\
\hline SP6 & $1.50 \times 10^{-03}$ & $1.76 \times 10^{-03}$ \\
\hline PKD2 & $1.90 \times 10^{-06}$ & $2.03 \times 10^{-03}$ \\
\hline UBE2E2 & $1.70 \times 10^{-04}$ & $2.13 \times 10^{-03}$ \\
\hline TNFRSF11A & $2.20 \times 10^{-09}$ & $2.41 \times 10^{-03}$ \\
\hline RBPMS2 & $4.90 \times 10^{-14}$ & $2.42 \times 10^{-03}$ \\
\hline SLC45A3 & $3.30 \times 10^{-02}$ & $3.76 \times 10^{-03}$ \\
\hline ANKRD6 & $4.50 \times 10^{-06}$ & $3.83 \times 10^{-03}$ \\
\hline EGR2 & $3.60 \times 10^{-02}$ & $3.89 \times 10^{-03}$ \\
\hline TMPRSS4 & $4.80 \times 10^{-03}$ & $4.44 \times 10^{-03}$ \\
\hline TTC7B & $1.40 \times 10^{-04}$ & $4.57 \times 10^{-03}$ \\
\hline INHBB & $1.10 \times 10^{-06}$ & $4.80 \times 10^{-03}$ \\
\hline LYPD1 & $7.70 \times 10^{-05}$ & $5.76 \times 10^{-03}$ \\
\hline C1orf216 & $1.00 \times 10^{-03}$ & $5.87 \times 10^{-03}$ \\
\hline CTHRC1 & $3.20 \times 10^{-02}$ & $6.03 \times 10^{-03}$ \\
\hline DHRS11 & $1.10 \times 10^{-02}$ & $6.35 \times 10^{-03}$ \\
\hline PBX3 & $4.40 \times 10^{-07}$ & $6.61 \times 10^{-03}$ \\
\hline PIK3R3 & $2.00 \times 10^{-03}$ & $7.17 \times 10^{-03}$ \\
\hline PCSK7 & $4.50 \times 10^{-06}$ & $8.36 \times 10^{-03}$ \\
\hline DFNA5 & $5.50 \times 10^{-06}$ & $9.16 \times 10^{-03}$ \\
\hline CATSPERB & $5.30 \times 10^{-03}$ & $9.93 \times 10^{-03}$ \\
\hline PPARG & $6.20 \times 10^{-05}$ & $1.06 \times 10^{-02}$ \\
\hline SLC44A3 & $4.60 \times 10^{-03}$ & $1.38 \times 10^{-02}$ \\
\hline STAMBPL1 & $2.20 \times 10^{-04}$ & $1.74 \times 10^{-02}$ \\
\hline ALPK1 & $6.80 \times 10^{-07}$ & $1.76 \times 10^{-02}$ \\
\hline SERAC1 & $5.70 \times 10^{-05}$ & $1.78 \times 10 \mathrm{v}$ \\
\hline BCAR3 & $1.60 \times 10^{-02}$ & $2.02 \times 10^{-02}$ \\
\hline TJP3 & $1.90 \times 10^{-03}$ & $2.20 \times 10^{-02}$ \\
\hline TMEM144 & $3.70 \times 10^{-06}$ & $2.22 \times 10^{-02}$ \\
\hline STARD5 & $8.40 \times 10^{-04}$ & $2.33 \times 10^{-02}$ \\
\hline BPNT1 & $8.20 \times 10^{-05}$ & $2.36 \times 10^{-02}$ \\
\hline CCDC92 & $9.00 \times 10^{-07}$ & $2.52 \times 10^{-02}$ \\
\hline RNF170 & $2.60 \times 10^{-03}$ & $2.58 \times 10^{-02}$ \\
\hline FBXL6 & $1.20 \times 10^{-03}$ & $2.83 \times 10^{-02}$ \\
\hline CASP7 & $6.70 \times 10^{-11}$ & $3.01 \times 10^{-02}$ \\
\hline RILPL1 & $1.30 \times 10^{-07}$ & $3.17 \times 10^{-02}$ \\
\hline KLHDC8B & $1.80 \times 10^{-07}$ & $3.34 \times 10^{-02}$ \\
\hline HOXC4 & $3.40 \times 10^{-04}$ & $3.62 \times 10^{-02}$ \\
\hline FAM83E & $1.10 \times 10^{-02}$ & $3.71 \times 10^{-02}$ \\
\hline MFSD9 & $2.20 \times 10^{-04}$ & $3.98 \times 10^{-02}$ \\
\hline ZNRF2 & $5.10 \times 10^{-07}$ & $4.01 \times 10^{-02}$ \\
\hline NMNAT1 & $2.50 \times 10^{-08}$ & $4.18 \times 10^{-02}$ \\
\hline BTNL3 & $5.40 \times 10^{-03}$ & $4.65 \times 10^{-02}$ \\
\hline F12 & $5.30 \times 10^{-05}$ & $4.96 \times 10^{-02}$ \\
\hline
\end{tabular}


Table III. Prognosis-associated clinical factors and the optimal prognosis-associated pathways in the Cox-PH model.

\begin{tabular}{|c|c|c|c|c|}
\hline Feature & Description & Coefficient & $\begin{array}{c}\text { Hazard } \\
\text { ratio }\end{array}$ & $\begin{array}{c}\text { P-value } \\
\text { (univariate } \\
\text { Cox-PH) }\end{array}$ \\
\hline \multirow[t]{16}{*}{ Pathway } & BIOCARTA_DEATH_PATHWAY & 0.115 & 1.966 & $8.73 \times 10^{-06}$ \\
\hline & BIOCARTA_DNAFRAGMENT_PATHWAY & 0.188 & 1.716 & $1.51 \times 10^{-05}$ \\
\hline & BIOCARTA_MITOCHONDRIA_PATHWAY & 0.614 & 3.156 & $4.62 \times 10^{-06}$ \\
\hline & BIOCARTA_PARKIN_PATHWAY & 0.285 & 1.737 & $2.06 \times 10^{-05}$ \\
\hline & KEGG_CHRONIC_MYELOID_LEUKEMIA & -0.029 & 0.398 & $1.36 \times 10^{-04}$ \\
\hline & KEGG_ENDOMETRIAL_CANCER & -0.299 & 0.260 & $1.02 \times 10^{-02}$ \\
\hline & KEGG_ERBB_SIGNALING_PATHWAY & -0.180 & 0.849 & $4.94 \times 10^{-03}$ \\
\hline & KEGG_FC_EPSILON_RI_SIGNALING_PATHWAY & -0.096 & 0.619 & $6.39 \times 10^{-03}$ \\
\hline & KEGG_FOCAL_ADHESION & -0.562 & 0.178 & $1.54 \times 10^{-02}$ \\
\hline & KEGG_JAK_STAT_SIGNALING_PATHWAY & -0.256 & 0.374 & $4.50 \times 10^{-06}$ \\
\hline & KEGG_LEUKOCYTE_TRANSENDOTHELIAL_MIGRATION & 0.344 & 1.031 & $2.22 \times 10^{-05}$ \\
\hline & KEGG_NATURAL_KILLLER_CELL_MEDIATED_CYTOTOXICITY & 0.024 & 1.062 & $1.33 \times 10^{-06}$ \\
\hline & KEGG_NICOTINATE_AND_NICOTINAMIDE_METABOLISM & 0.359 & 1.569 & $8.86 \times 10^{-05}$ \\
\hline & KEGG_PATHWAYS_IN_CANCER & -0.143 & 0.332 & $1.52 \times 10^{-06}$ \\
\hline & KEGG_PPAR_SIGNALING_PATHWAY & -0.135 & 0.835 & $2.07 \times 10^{-04}$ \\
\hline & KEGG_TIGHT_JUNCTION & 0.959 & 1.353 & $2.50 \times 10^{-13}$ \\
\hline \multirow[t]{4}{*}{ Clinical factor } & Recurrence & 2.074 & 2.574 & $2.00 \times 10^{-16}$ \\
\hline & Pathologic_N & 0.165 & 1.956 & $3.97 \times 10^{-13}$ \\
\hline & Pathologic_stage & 0.233 & 2.215 & $6.66 \times 10^{-16}$ \\
\hline & MLH1 mutation & 0.024 & 2.027 & $2.69 \times 10^{-03}$ \\
\hline
\end{tabular}

Cox-PH, Cox-proportional hazards; KEGG, Kyoto Encyclopedia of Genes and Genomes; MLH1, mutL homolog 1.



Figure 2. Clustering heatmap based on 50 prognosis-associated genes. The first line below the sample tree represents group 1 (black) and group 2 (white); the second line indicates samples negative for MLH1 mutation (blue) and samples positive for MLH1 mutation (red); the third line represents pathological stages I (light orange), II (orange), III (orange-red) and IV (maroon); the fourth line represents Pathological N0 (light pink), N1 (dark red), N2 (light blue) and N3 (dark blue) samples; the fifth line represents recurrent samples (bright blue) and non-recurrent samples (purple) (white indicates samples without recurrence information). MLH1, mutL homolog 1. 
A

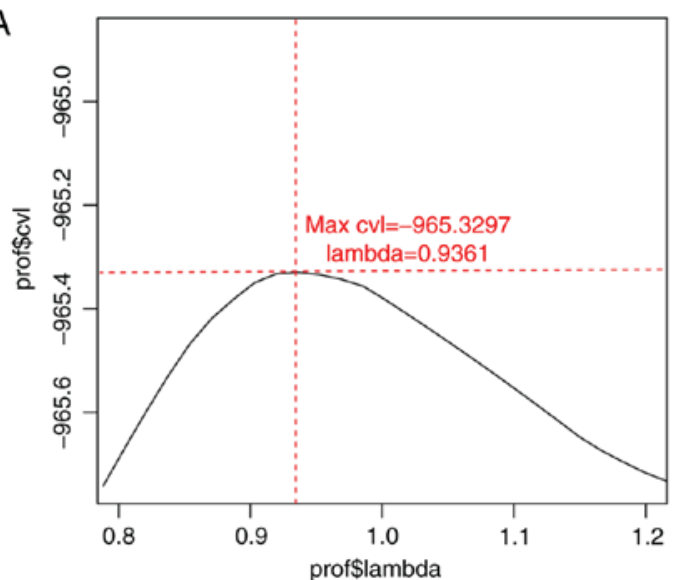

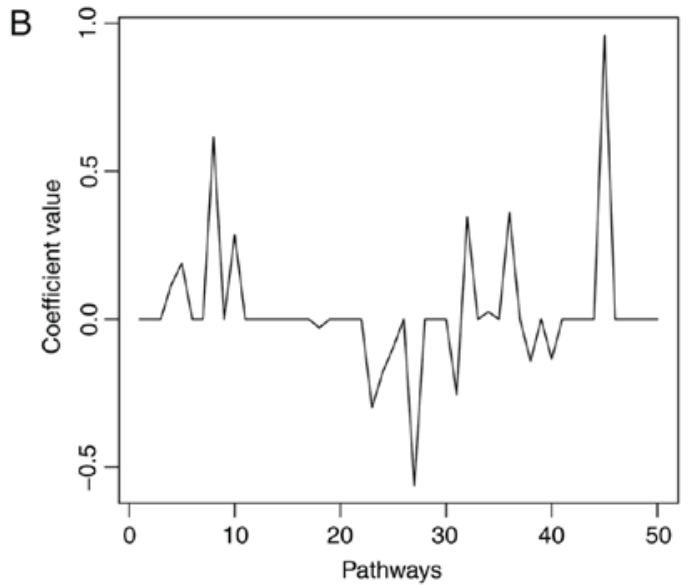

Figure 3. Selection of prognosis associated pathways. (A) Curve showing the parameter 'lambda' screened by cvl and (B) the coefficient distribution diagram of the optimal prognosis-associated pathways (B). cvl, cross-validation likelihood.

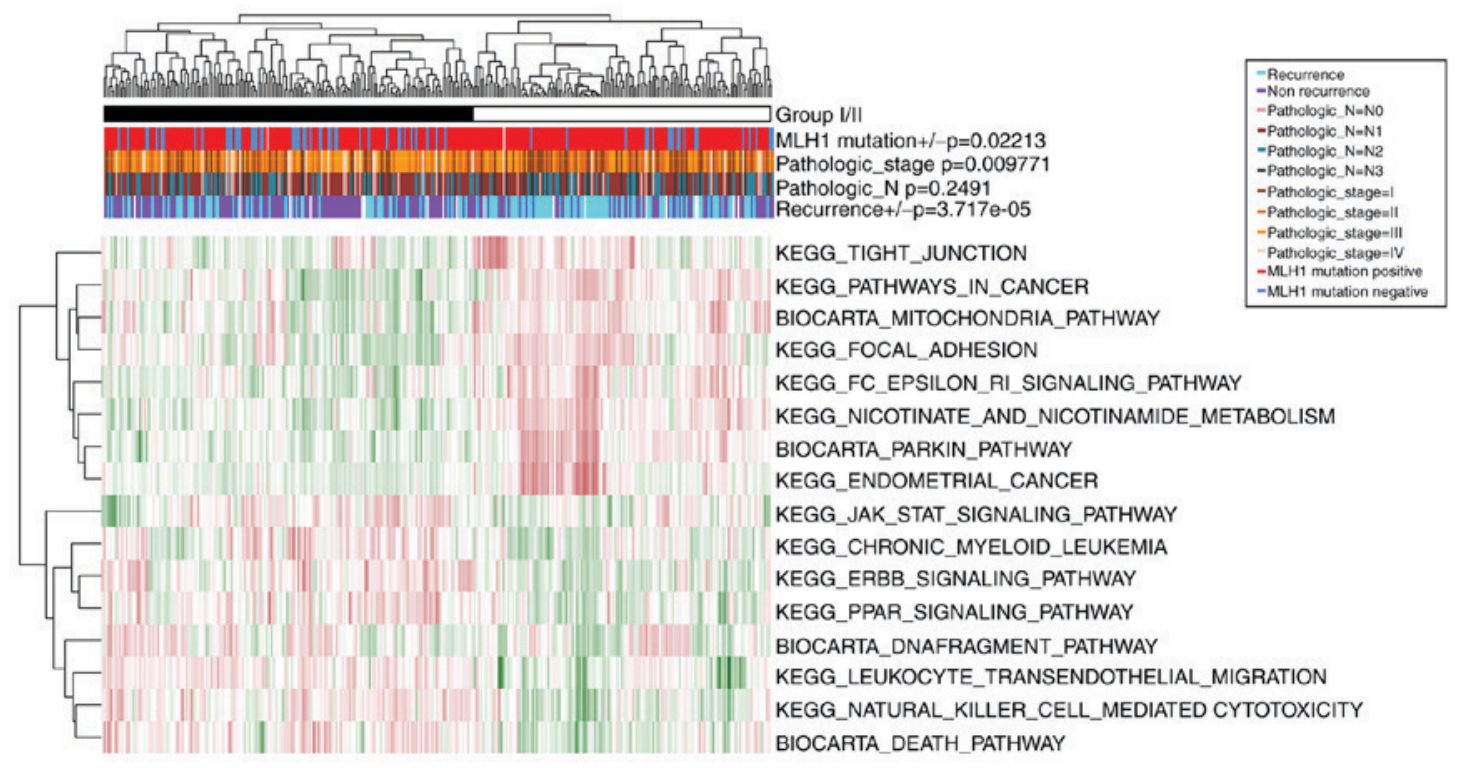

Figure 4. Bidirectional hierarchical clustering heatmap based on the 16 optimal prognosis-associated pathways. The first line below the sample tree represents groups I (black) and II (white); the second line indicates MLH1 mutation negative samples (blue) and MLH1 mutation positive samples (red); the third line represents pathological stages I (light orange), II (orange), III (orange-red) and IV (maroon); the fourth line represents pathologic N0 (light pink), N1 (dark red), N2 (light blue) and N3 (dark blue) samples; the fifth line represents recurrent samples (bright blue) and non-recurrent samples (purple) (white indicates samples without recurrence information). MLH1, mutL homolog 1; KEGG, Kyoto Encyclopedia of Genes and Genomes.

$134 \mathrm{GC}$ samples), according to the clustering analysis of the PDS matrix of the 16 optimal prognosis-associated pathways (Fig. 4). Similarly, there were significant differences in recurrence $\left(\mathrm{P}=3.72 \times 10^{-5}\right)$, Pathologic_stage $\left(\mathrm{P}=9.77 \times 10^{-3}\right)$, and MLH1 mutation $\left.\mathrm{P}=2.21 \times 10^{-2}\right)$ between these two groups. However, no notable difference was observed in Pathologic_N $\left(\mathrm{P}=2.49 \times 10^{-1}\right)$. Thus, group I had less recurrence, lower tumor stage and more MLH1 mutations compared with group II.

Construction and validation of risk prediction models. The pathway-based risk prediction model was constructed and the PI score of each sample was obtained. Subsequently, the samples in the training set were divided into high- and low-risk groups (Fig. 5). Compared with the high-risk group, the low-risk group had a longer overall survival (OS) time (59.85 29.87 months vs. $41.36 \pm 29.65$ months) and recurrence-free survival (RFS) time (44.65 \pm 28.99 months vs. $30.00 \pm 28.35 \mathrm{~m}$ onths) (Fig. 5A and B). The risk groups exhibited significant correlations with OS time $\left(\mathrm{P}=4.90 \times 10^{-7}\right.$; Fig. 5A) and RFS time $\left(\mathrm{P}=2.44 \times 10^{-7}\right.$; Fig. 5B). Furthermore, the area under the receiver operating characteristic (AUROC) values of OS and RFS were 0.8554 and 0.809 , respectively (Fig. 5E). In the validation set, the low-risk group additionally had a longer OS time $(23.41 \pm 22.06$ vs. $13.56 \pm 7.46$ months $)$ and RFS time $(2.33 \pm 22.14$ vs. $13.06 \pm 7.52$ months) compared with the high-risk group (Fig. 5C and D). The risk groups had significant correlations with OS time ( $\mathrm{P}=1.15 \times 10^{-4}$; Fig. $\left.5 \mathrm{C}\right)$ and RFS time ( $\mathrm{P}=2.62 \times 10^{-6}$; Fig. 5D). Furthermore, the AUROC values of OS and RFS were 0.733 and 0.7559 , respectively (Fig. 5E). These results indicated that the pathway-based risk prediction model was able to predict the consistent sample risk in the training set and the validation set. 
A

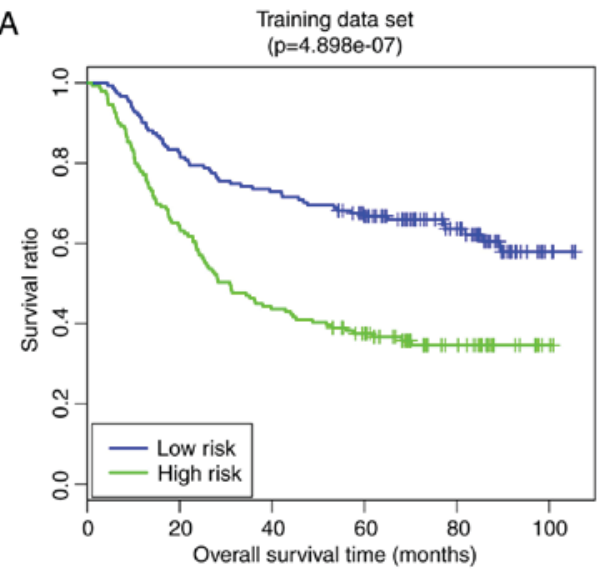

C

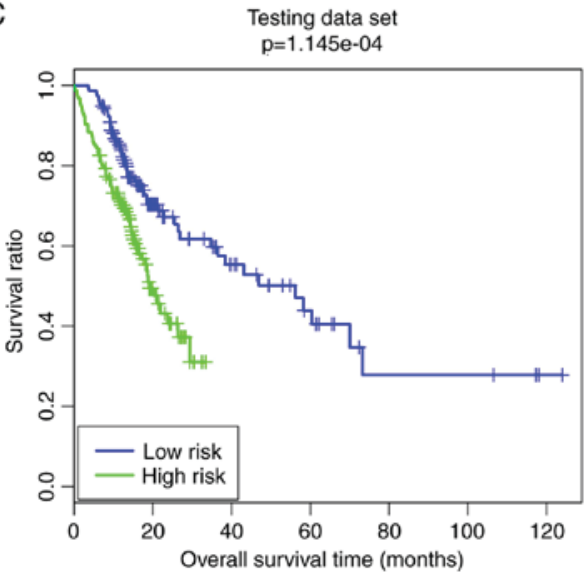

B
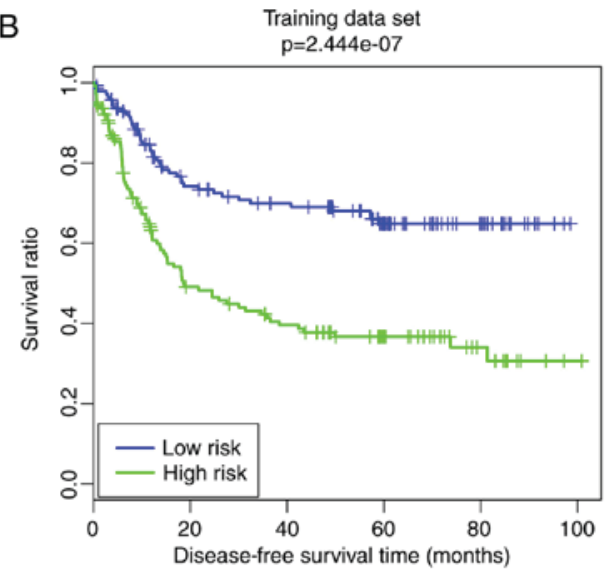

D

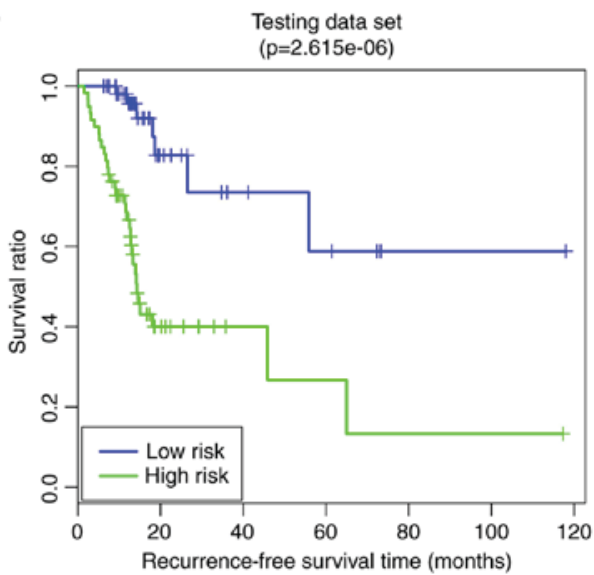

E

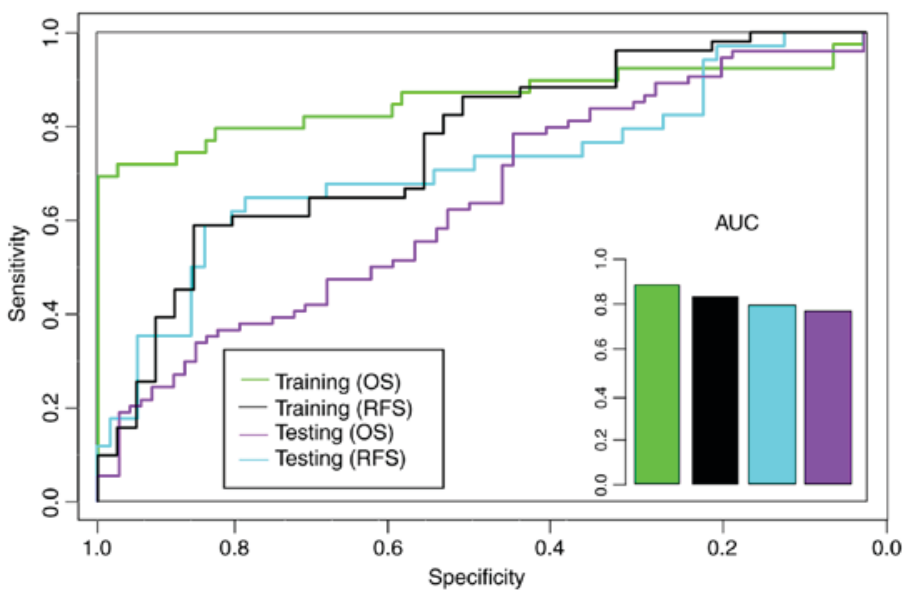

Figure 5. KM survival curves and ROC curves based on the pathway-based risk prediction model. (A) The KM survival curve illustrating the OS time in the training set; (B) the KM survival curve illustrating the RFS time in the training set; (C) the KM survival curve illustrating the OS time in the validation set; (D) the KM survival curve illustrating the RFS time in the validation set; (E) the ROC curves illustrating the OS and RFS separately in the training and validation sets. KM, Kaplan-Meier; ROC, receiver operating characteristic; AUC, area under the curve; OS, overall survival; RFS, recurrence-free survival.

Using the Cox-PH model, 10 optimal prognosis-associated genes were identified (Table IV). The gene-based risk prediction model was built, and the samples in training set were divided into high- and low-risk groups (Fig. 6). In the training set, the low-risk group had a longer OS time (58.20 \pm 31.09 vs. $42.99 \pm 29.96$ months) and RFS time (38.31 \pm 30.70 vs. $29.13 \pm 28.35$ months) compared with the high-risk group (Fig. 6A and B). The risk groups had significant correlations with OS time $\left(\mathrm{P}=3.06 \times 10^{-4}\right.$; Fig. $\left.6 \mathrm{~A}\right)$ and RFS time ( $\mathrm{P}=3.62 \times 10^{-4}$; Fig. $\left.6 \mathrm{~B}\right)$. The AUROC values of OS and RFS were 0.7966 and 0.7129 , respectively (Fig. 6E).
In the validation set, the low-risk group had a longer OS time (23.85 \pm 23.02 vs. $18.18 \pm 21.58$ months) and RFS time (18.95 \pm 17.73 vs. $18.78 \pm 19.48$ months) compared with the high-risk group (Fig. 6C and D). The risk groups had a significant correlation with OS time ( $\mathrm{P}=4.88 \times 10^{-2}$; Fig. $\left.6 \mathrm{C}\right)$, although not with RFS time ( $\mathrm{P}=8.50 \times 10^{-1}$; Fig. 6D). The AUROC values of OS and RFS were 0.6969 and 0.6453 , respectively (Fig. 6E). These findings suggested that the gene-based risk prediction model was not able to be completely verified in the validation set. In this way, the pathway-based risk prediction model outperformed the gene-based risk prediction model. 

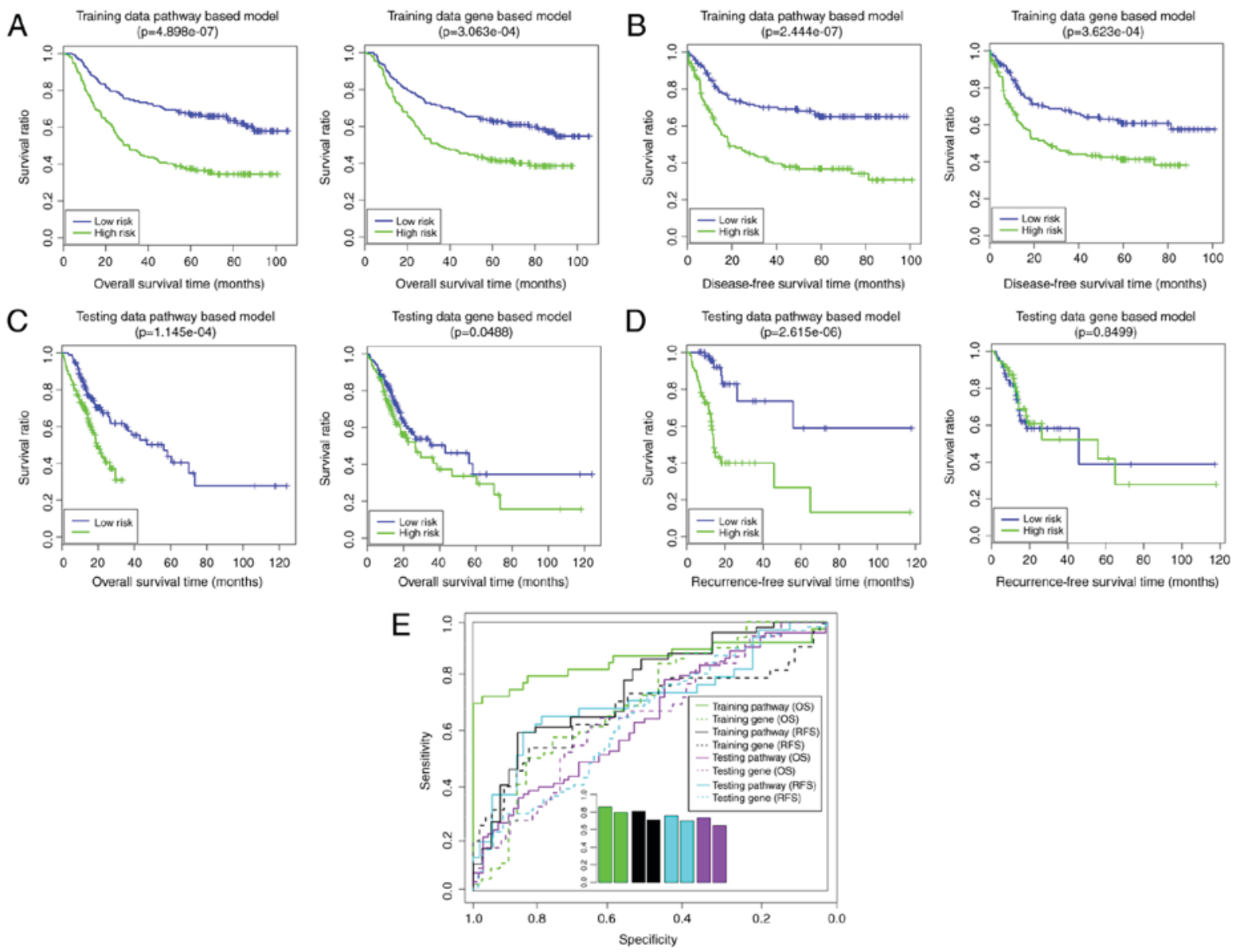

Figure 6. KM survival curves and ROC curves based on the pathway-based risk prediction model and the gene-based risk prediction model. (A) The KM survival curves illustrating the OS time in the training set; (B) the KM survival curves illustrating the RFS time in the training set; (C) the KM survival curves illustrating the OS time in the validation set; (D) the KM survival curves illustrating the RFS time in the validation set; (E) the ROC curves illustrating the OS and RFS separately in the training and validation sets. KM, Kaplan-Meier; ROC, receiver operating characteristic; OS, overall survival; RFS, recurrence-free survival.

Table IV. Optimal prognosis-associated genes $(\mathrm{n}=10)$ identified by the Cox-PH model.

\begin{tabular}{lccc}
\hline Gene & Coefficient & $\begin{array}{c}\text { Hazard } \\
\text { ratio }\end{array}$ & $\begin{array}{c}\text { P-value } \\
\text { (univariate } \\
\text { Cox-PH) }\end{array}$ \\
\hline CATSPERB & -0.935 & 0.970 & $1.09 \times 10^{-04}$ \\
CDC42EP5 & 0.176 & 0.551 & $5.42 \times 10^{-04}$ \\
CTHRC1 & -0.457 & 0.761 & $2.13 \times 10^{-03}$ \\
DHRS11 & -0.160 & 1.232 & $2.42 \times 10^{-03}$ \\
EGR2 & -0.196 & 0.283 & $3.89 \times 10^{-03}$ \\
INHBB & 0.127 & 2.043 & $4.80 \times 10^{-03}$ \\
RAB11FIP4 & -0.224 & 1.058 & $6.03 \times 10^{-03}$ \\
RBPMS2 & 0.322 & 3.517 & $6.35 \times 10^{-03}$ \\
STAMBPL1 & -0.244 & 0.274 & $9.93 \times 10^{-03}$ \\
UBE2E2 & 0.303 & 4.114 & $1.74 \times 10^{-02}$ \\
\hline
\end{tabular}

Cox-PH, Cox-proportional hazards; CATSPERB, cation channel sperm associated auxiliary subunit $\beta$; CDC42EP5, CDC42 effector protein 5; CTHRC1, collagen triple helix repeat containing 1; DHRS11, dehydrogenase/reductase 11; EGR2, early growth response 2; INHBB, inhibin $\beta$ B subunit; RAB11FIP4, RAB11 family interacting protein 4 ; RBPMS2, RNA binding protein mRNA processing factor 2; STAMBPL1, STAM binding protein-like 1; UBE2E2, ubiquitin conjugating enzyme E2 E2.
A clinical factor-based risk prediction model was constructed based on the four prognosis-associated clinical factors. In the training set, the low-risk group had a longer OS time (63.55 \pm 26.36 vs. $6.41 \pm 30.24$ months) and RFS time (48.21 \pm 28.19 vs. $22.55 \pm 25.59$ months) compared with the high-risk group (Fig. 7A and B). The risk groups had significant correlations with OS time $\left(\mathrm{P}=7.11 \times 10^{-15}\right.$; Fig. $\left.7 \mathrm{~A}\right)$ and RFS time $\left(\mathrm{P}=1.11 \times 10^{-16}\right.$; Fig. $\left.7 \mathrm{~B}\right)$. In the validation set, the low-risk group had a longer OS time $(20.06 \pm 16.33$ vs. $18.66 \pm 17.75$ months) and RFS time $(22.52 \pm 24.23$ vs. $16.93 \pm 10.74$ months) compared with the high-risk group (Fig. 7C and D). The risk group had significant correlations with OS time $\left(\mathrm{P}=1.51 \times 10^{-2}\right.$; Fig; 7C) and RFS time ( $\mathrm{P}=1.56 \times 10^{-12}$; Fig. 7D).

Finally, the comprehensive risk prediction model based on the optimal prognosis-associated pathways and the prognosis-associated clinical factors was constructed. In the training set, the low-risk group had longer OS time (64.34 \pm 25.33 vs. $36.76 \pm 30.09$ months) and RFS time (50.33 \pm 26.98 vs. $24.05 \pm 25.81$ months) compared with the high-risk group (Fig. 8A and B). The risk groups had significant correlations with OS time $\left(\mathrm{P}=1.18 \times 10^{-14}\right.$; Fig. $\left.8 \mathrm{~A}\right)$ and RFS time $\left(\mathrm{P}=2.00 \times 10^{-16}\right.$; Fig. $\left.8 \mathrm{~B}\right)$. In the validation set, the low risk group had a longer OS time ( $21.37 \pm 16.41$ vs. $18.35 \pm 17.68$ months) and RFS time ( $22.01 \pm 20.32$ vs. $19.35 \pm 17.73$ months) compared 
A

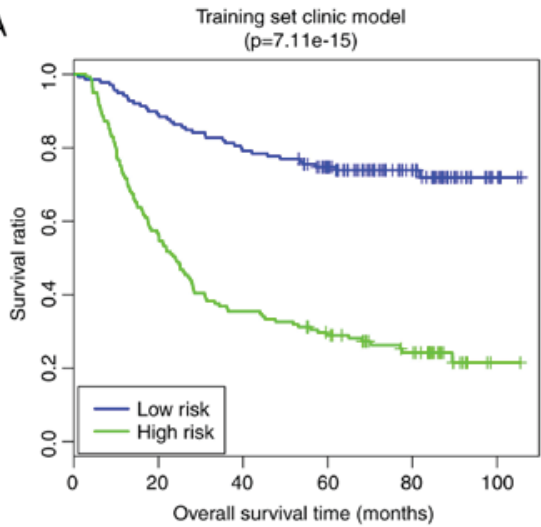

C

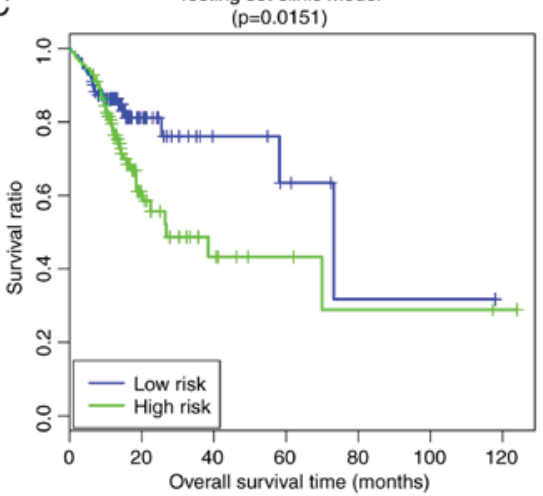

B

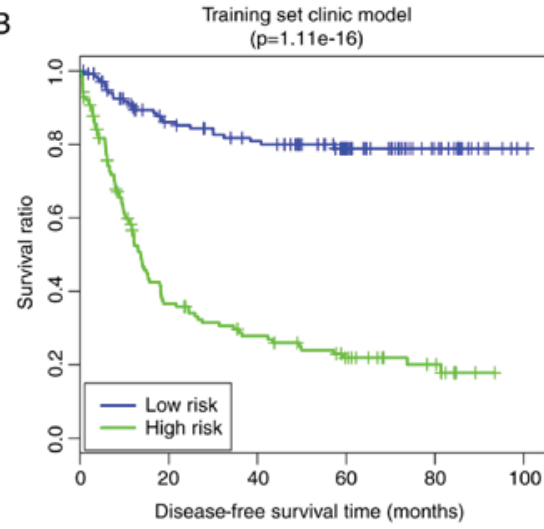

D

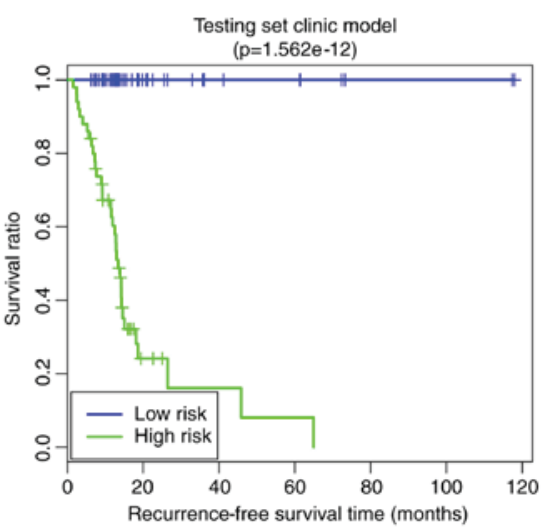

Figure 7. KM survival curves based on the clinical factor-based risk prediction model. (A) The KM survival curve illustrating the OS time in the training set; (B) the KM survival curve illustrating the RFS time in the training set; (C) the KM survival curve illustrating the OS time in the validation set; (D) the KM survival curve illustrating the RFS time in the validation set. Blue and green represent low- and high-risk groups, respectively. KM, Kaplan-Meier; OS, overall survival; RFS, recurrence-free survival.

A

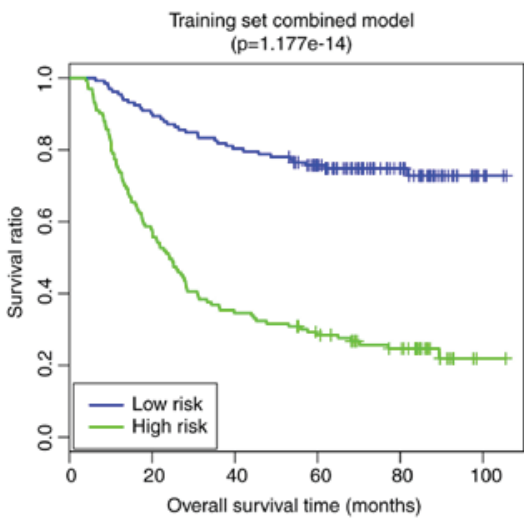

$\mathrm{C}$

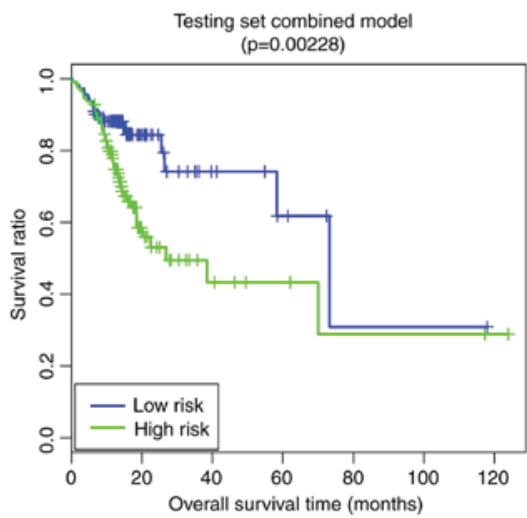

B

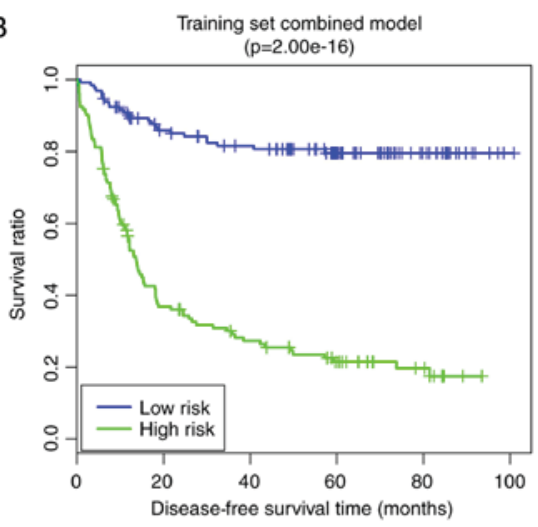

D

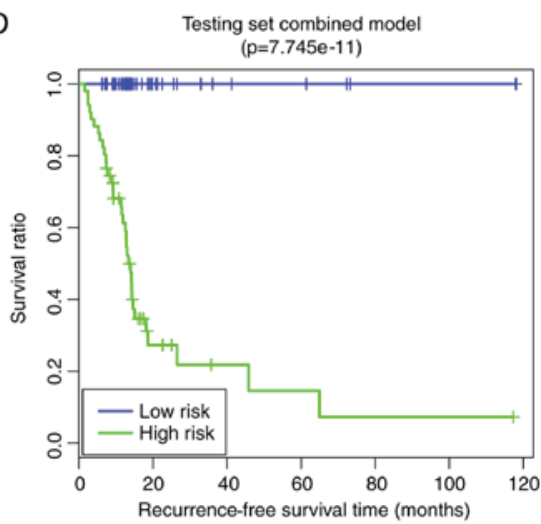

Figure 8. KM survival curves based on the pathway and clinical factor-based risk prediction model. (A) KM survival curve illustrating the OS time in the training set; (B) the KM survival curve illustrating the RFS time in the training set; (C) the KM survival curve illustrating the OS time in the validation set; (D) the KM survival curve illustrating the RFS time in the validation set. Blue and green represent low- and high-risk groups, respectively. KM, Kaplan-Meier; OS, overall survival; RFS, recurrence-free survival. 
with the high-risk group (Fig. 8C and D). The risk groups had significant correlations with OS time $\left(\mathrm{P}=2.28 \times 10^{-3}\right.$; Fig. $\left.8 \mathrm{C}\right)$ and RFS time $\left(\mathrm{P}=7.75 \times 10^{-11}\right.$; Fig. $\left.8 \mathrm{D}\right)$. The pathway- and clinical-factor-based risk prediction model may have better prognostic accuracy, since it had better robustness and more significant $\mathrm{P}$-values.

\section{Discussion}

In the present study, the gene expression profiles of the GSE62254 downloaded from GEO served as the training set and another mRNA-sequencing dataset obtained from TCGA served as the validation set. Although huge and heterogeneous collections frequently dilute specific results and favor secondary effects, these two datasets were independently standardized to partially eliminate the technology bias in systematic measurement. The 382 overlapping DEGs were screened for further analysis, including 268 upregulated genes and 114 downregulated genes. In the present study, 50 prognosis-associated genes and four prognosis-associated clinical factors (including Pathologic_N, Pathologic_stage, MLH1 mutation and recurrence) were identified. Based on the prognosis-associated genes, the samples in GSE62254 were divided into group 1 and group 2. The present results demonstrated that group 1 had less recurrence, less lymphatic metastasis, lower tumor stage and more MLH1 mutations compared with group 2. Using the Cox-PH model, 16 optimal prognosis-associated pathways (including the mitochondrial pathway and the JAK-STAT signaling pathway, involving CASP7, MYH14, NMNAT1, PIK3R3, PPARG, TJP3, CATSPERB, CDC42EP5, CTHRC1 and DHRS11) were selected. Similarly, the samples were divided into group I and II based on the 16 optimal prognosis-associated pathways. Group I was observed to have less recurrence, lower tumor stage and more MLH1 mutations compared with group II.

The expression levels of CASP2, CASP6 and CASP7 are decreased in GC cells, which may be associated with the pathogenesis of GC (31). CASP7 in the apoptosis pathway functions as a critical mediator and executor, and its potential functional variants may increase the risk of GC (32). Upregulated PIK3R3 was demonstrated to contribute to cell cycle progression and cell proliferation, indicating that PIK3R3 may be a promising target for the treatment of GC (33). The phosphatidylinositol 3-kinase/RAC- $\alpha$ serine/threonine-protein kinase/serine/threonine-protein kinase mTOR signaling pathway is considered to have been implicated in the mechanisms of GC and contributes to the identification of potential therapeutic targets for the disease $(34,35)$. Optimal prognosis-associated pathways may serve important roles in the pathogenesis of GC via CASP7 and PIK3R3.

PPARG, plasma gastrin and proinflammatory cytokines have been reported to be correlated with GC development, and PPARG agonists have the potential to be used for cancer therapy $(36,37)$. PPARG may suppress the proliferation and migration of GC cells by inhibiting enabled homolog and telomerase reverse transcriptase expression, thus PPARG may serve as a therapeutic target for GC (38). CTHRC1 expression may be regulated by transforming growth factor- $\beta 1$ and promoter demethylation, and high levels of CTHRC1 expression promote the invasion and metastasis of tumor cells during gastric carcinogenesis $(39,40)$. The upregulated expression of CTHRC1 may independently predict the disease-free survival and overall survival of patients with $\mathrm{GC}$, demonstrating that the high levels of expression of CTHRC1 are associated with the progression and prognosis of GC (41). These suggested that the optimal prognosis-associated pathways may be associated with the development and progression of GC via PPARG and CTHRC1.

Via a mitochondrial pathway, juglone has been reported to be able to induce the apoptosis of GC SGC-7901 cells (42). $H$. pylori infection leads to activation of CASP3 and CASP9, and to apoptosis in GC cells, and the mitochondrial pathway may be important to $H$. pylori-induced apoptosis (43). CyclinB1 expression may be downregulated by fucoxanthin in human GC MGC-803 cells, in which the JAK-STAT signaling pathway serves an important role (44). The mitochondrial pathway and JAK-STAT signaling pathway may be involved in the mechanisms of GC. A previous study demonstrated that MLH1 methylation status and CpG island methylator phenotype may be suitable prognostic biomarkers for patients with GC (45). Checkpoint with forkhead and ring finger domains methylation may be considered to be a docetaxel-sensitive marker, and MLH1 methylation is correlated with oxaliplatin resistance in patients with GC (46). These findings indicated that MLH1 mutation might serve as a prognostic biomarker for GC. Taken together, the prognosis-associated genes involved in the optimal-prognosis-associated pathways in the present prognosis model are promising targets for therapeutic intervention.

In the present study, the pathway-based risk prediction model and clinical-factor-based risk prediction model outperformed the gene-based risk prediction model. Although the gene-based risk prediction model had acceptable results in the training set (OS: $\mathrm{P}=3.06 \times 10^{-4}$, $\mathrm{AUC}=0.7966$; RFS: $\mathrm{P}=3.62 \times 10^{-4}$, $\mathrm{AUC}=0.7129)$, this model was not able to be completely verified in the validation set $\left(\mathrm{OS}: \mathrm{P}=4.88 \times 10^{-2}, \mathrm{AUC}=0.6969\right.$; RFS: $P>0.05)$. The results of the pathway-based risk prediction model in the training set (OS: $\mathrm{P}=4.90 \times 10^{-7}, \mathrm{AUC}=0.8554$; RFS: $\left.\mathrm{P}=2.44 \times 10^{-7}, \mathrm{AUC}=0.809\right)$ and in the validation set $(\mathrm{OS}$ : $\mathrm{P}=1.15 \times 10^{-4}$, $\mathrm{AUC}=0.733$; RFS: $\mathrm{P}=2.62 \times 10^{-6}, \mathrm{AUC}=0.7559$ ) indicated that this model had good performance. Furthermore, the clinical factor-based risk prediction model (training set, OS: $\mathrm{P}=7.11 \times 10^{-15}$, RFS: $\mathrm{P}=1.11 \times 10^{-16}$; validation set, OS: $\mathrm{P}=1.51 \times 10^{-2}$, RFS: $\left.\mathrm{P}=1.56 \times 10^{-12}\right)$ improved the P-values of prognosis prediction, rendering them higher compared with those of the pathway-based risk prediction model. The comprehensive risk prediction model, based on the optimal prognosis-associated pathways and the prognosis-associated clinical factors, yielded good predictive results (training set, OS: $\mathrm{P}=1.18 \times 10^{-14}$, RFS: $\mathrm{P}=2.00 \times 10^{-16}$; validation set, OS: $\mathrm{P}=2.28 \times 10^{-3}$, RFS: $\left.\mathrm{P}=7.75 \times 10^{-11}\right)$. In this way, the pathway and clinical factor-based risk prediction model may be suitable for predicting the prognosis of patients with GC.

In conclusion, 50 prognosis-associated genes, 16 optimal prognosis-associated pathways and four prognosis-associated clinical factors were identified. The pathway and clinical factor-based risk prediction model might be suitable for predicting the prognosis of GC patients. The prognosis-associated genes involved in the optimal prognosis-associated pathways in the present prognostic model (including CASP7, PIK3R3, PPARG, 
CTHRC1 and MLH1) are promising targets for therapeutic intervention. However, further study is required to validate the prognostic prediction model, based on the optimal prognosis-associated pathways and the prognosis-associated clinical factors, in an independent patient cohort with gastric cancer.

\section{Acknowledgements}

Not applicable.

\section{Funding}

No funding was received.

\section{Availability of data and materials}

The datasets used and/or analyzed during the current study are available from the corresponding author on reasonable request.

\section{Authors' contributions}

MN and KY conceived and designed the study. JY and LB designed and performed data analyses. TH collected the data and wrote the manuscript. DD participated in study design and organized the literature. All authors read and approved the final manuscript.

\section{Ethical approval and consent to participate}

Not applicable.

\section{Consent for publication}

Not applicable.

\section{Competing interests}

The authors declare that they have no competing interests.

\section{References}

1. Hartgrink HH, Jansen EP, van Grieken NC and van de Velde CJ: Gastric cancer. Lancet 374: 477-490, 2009.

2. Sim F and Mckee M: Issues in Public Health. Open University Press, 2011.

3. World Health Organization: World Cancer Report, 2014.

4. Chang AH and Parsonnet J: Role of bacteria in oncogenesis. Clin Microbiol Rev 23: 837-857, 2010.

5. Choi YY, An JY, Kim HI, Cheong JH, Hyung WJ and Noh SH: Current practice of gastric cancer treatment. Chin Med J 127: $547-553,2014$

6. Raymond W and Ruddon RW: Cancer Biology. 4th edition. Oxford University Press, Oxford, pp791, 2007.

7. Orditura M, Galizia G, Sforza V, Gambardella V, Fabozzi A, Laterza MM, Andreozzi F, Ventriglia J, Savastano B, Mabilia A, et al: Treatment of gastric cancer. World J Gastroenterol 20: 1635-1649, 2014

8. Jian-bo X, Hui W, Yu-long H, Chang-hua Z, Long-juan Z, Shi-rong C and Wen-hua Z: Astrocyte-elevated gene-1 overexpression is associated with poor prognosis in gastric cancer. Med Oncol 28: 455-462, 2011.

9. Liang L, Li L, Tian J, Lee SO, Dang Q, Huang CK, Yeh S, Erturk E, Bushinsky D, Chang LS, et al: Androgen receptor enhances kidney stone- $\mathrm{CaOx}$ crystal formation via modulation of oxalate biosynthesis \& oxidative stress. Mol Endocrinol 28: 1291-1303, 2014
10. Gomez-Martin C, Plaza JC, Pazo-Cid R, Salud A, Pons F, Fonseca P, Leon A, Alsina M, Visa L, Rivera F, et al: Level of HER2 gene amplification predicts response and overall survival in HER2-positive advanced gastric cancer treated with trastuzumab. J Clin Oncol 31: 4445-4452, 2013.

11. Wang DD, Chen YB, Pan K, Wang W, Chen SP, Chen JG, Zhao JJ, Lv L, Pan QZ, Li YQ, et al: Decreased expression of the ARIDIA gene is associated with poor prognosis in primary gastric cancer. PLoS One 7: e40364, 2012.

12. Yan HB, Wang XF, Zhang Q, Tang ZQ, Jiang YH, Fan HZ, Sun YH, Yang PY and Liu F: Reduced expression of the chromatin remodeling gene ARIDIA enhances gastric cancer cell migration and invasion via downregulation of E-cadherin transcription. Carcinogenesis 35: 867-876, 2014.

13. Okugawa Y, Tanaka K, Inoue Y, Kawamura M, Kawamoto A, Hiro J, Saigusa S, Toiyama Y, Ohi M, Uchida K, et al: Brain-derived neurotrophic factor/tropomyosin-related kinase B pathway in gastric cancer. Br J Cancer 108: 121-130, 2013.

14. Santoro R, Carboni F, Lepiane P, Ettorre GM and Santoro E: Clinicopathological features and prognosis of gastric cancer in young European adults. Br J Surg 94: 737-742, 2007.

15. Shimada H, Noie T, Ohashi M, Oba K and Takahashi Y: Clinical significance of serum tumor markers for gastric cancer: A systematic review of literature by the Task Force of the Japanese Gastric Cancer Association. Gastric Cancer 17: 26-33, 2014.

16. Huang S, Yee C, Ching T, Yu H and Garmire LX: A novel model to combine clinical and pathway-based transcriptomic information for the prognosis prediction of breast cancer. PLoS Comput Biol 10: e1003851, 2014.

17. Huang S, Chong N, Lewis NE, Jia W, Xie G and Garmire LX: Novel personalized pathway-based metabolomics models reveal key metabolic pathways for breast cancer diagnosis. Genome Med 8: 34, 2016.

18. Cristescu R, Lee J, Nebozhyn M, Kim KM, Ting JC, Wong SS, Liu J, Yue YG, Wang J, Yu K, et al: Molecular analysis of gastric cancer identifies subtypes associated with distinct clinical outcomes. Nat Med 21: 449-456, 2015.

19. Woo HG, Park ES, Cheon JH, Kim JH, Lee JS, Park BJ, Kim W, Park SC, Chung YJ, Kim BG, et al: Gene expression-based recurrence prediction of hepatitis B virus-related human hepatocellular carcinoma. Clin Cancer Res 14: 2056-2064, 2008.

20. Irizarry RA, Bolstad BM, Collin F, Cope LM, Hobbs B and Speed TP: Summaries of Affymetrix GeneChip probe level data. Nucleic Acids Res 31: e15, 2003.

21. R Core Team: R: A language and environment for statistical computing. Vienna, Austria: The R Foundation for Statistical Computing. http://www.R-project.org/. 2017.

22. Kruschke JK: Bayesian estimation supersedes the $t$ test. J Exp Psychol Gen 142: 573-603, 2013.

23. Nagarajan N and Keich U: Reliability and efficiency of algorithms for computing the significance of the Mann-Whitney test. Comput Stat 24: 605, 2009.

24. Therneau T: A package for survival analysis in S. R package version 2: 37-37, 2014

25. Farinelli A, Bicego M, Bistaffa F and Ramchurn SD: A hierarchical clustering approach to large-scale near-optimal coalition formation with quality guarantees. Eng Appl Art Intell 59: 170-185, 2017.

26. Kolde R: Pheatmap: Pretty Heatmaps [Software], 2015.

27. Subramanian A, Tamayo P, Mootha VK, Mukherjee S, Ebert BL, Gillette MA, Paulovich A, Pomeroy SL, Golub TR, Lander ES and Mesirov JP: Gene set enrichment analysis: A knowledge-based approach for interpreting genome-wide expression profiles. Proc Natl Acad Sci USA 102: 15545-15550, 2005.

28. Drier Y, Sheffer M and Domany E: Pathway-based personalized analysis of cancer. Proc Natl Acad Sci USA 110: 6388-6393, 2013.

29. Goeman JJ: L1 penalized estimation in the Cox proportional hazards model. Biom J 52: 70-84, 2010.

30. Bachoc F: Cross validation and maximum likelihood estimations of hyper-parameters of Gaussian processes with model misspecification. Comput Stat Data Anal 66: 55-69, 2013.

31. Yoo NJ, Lee JW, Kim YJ, Soung YH, Kim SY, Nam SW, Park WS Lee JY and Lee SH: Loss of caspase-2, -6 and -7 expression in gastric cancers. APMIS 112: 330-335, 2004.

32. Wang MY, Zhu ML, He J, Shi TY, Li QX, Wang YN, Li J, Zhou XY, Sun MH, Wang XF, et al: Potentially functional polymorphisms in the CASP7 gene contribute to gastric adenocarcinoma susceptibility in an eastern Chinese population. PLoS One 8: e74041, 2013. 
33. Zhou J, Chen GB, Tang YC, Sinha RA, Wu Y, Yap CS, Wang G, Hu J, Xia X, Tan P, et al: Genetic and bioinformatic analyses of the expression and function of PI3K regulatory subunit PIK3R3 in an Asian patient gastric cancer library. BMC Med Genomics 5: 34, 2012.

34. Fang WL, Huang KH, Lan YT, Lin CH, Chang SC, Chen MH, Chao Y, Lin WC, Lo SS, Li AF, et al: Mutations in PI3K/AKT pathway genes and amplifications of PIK3CA are associated with patterns of recurrence in gastric cancers. Oncotarget 7 : 6201-6220, 2016.

35. Matsuoka T and Yashiro M: The role of PI3K/Akt/mTOR signaling in gastric carcinoma. Cancers 6: 1441-1463, 2014.

36. Konturek PC, Kania J, Kukharsky V, Raithel M, Ocker M, Rembiasz K, Hahn EG and Konturek SJ: Implication of peroxisome proliferator-activated receptor gamma and proinflammatory cytokines in gastric carcinogenesis: Link to Helicobacter pylori-infection. J Pharmacol Sci 96: 134-143, 2004.

37. Konturek PC, Kania J, Konturek JW, Nikiforuk A, Konturek SJ and Hahn EG: H. pylori infection, atrophic gastritis, cytokines, gastrin, COX-2, PPAR gamma and impaired apoptosis in gastric carcinogenesis. Med Sci Monit 9: SR53-SR66, 2003.

38. Guo F, Ren X, Dong Y, Hu X, Xu D, Zhou H, Meng F, Tian W and Zhao Y: Constitutive expression of PPAR $\gamma$ inhibits proliferation and migration of gastric cancer cells and down-regulates Wnt/ $\beta$-Catenin signaling pathway downstream target genes TERT and ENAH. Gene 584: 31-37, 2016.

39. Wang P, Wang YC, Chen XY, Shen ZY, Cao H, Zhang YJ Yu J, Zhu JD, Lu YY and Fang JY: CTHRCl is upregulated by promoter demethylation and transforming growth factor- $\beta 1$ and may be associated with metastasis in human gastric cancer. Cancer Sci 103: 1327-1333, 2012.

40. Yu J, Feng J, Zhi X, Tang J, Li Z, Xu Y, Yang L, Hu Z and Xu Z: Let-7b inhibits cell proliferation, migration, and invasion through targeting Cthrc1 in gastric cancer. Tumour Biol 36: 3221-3229, 2015.
41. Gu L, Liu L, Zhong L, Bai Y, Sui H, Wei X, Zhang W, Huang P, Gao D, Kong Y and Lou G: Cthrc1 overexpression is an independent prognostic marker in gastric cancer. Hum Pathol 45: 1031-1038, 2014.

42. Ji YB, Qu ZY and Zou X: Juglone-induced apoptosis in human gastric cancer SGC-7901 cells via the mitochondrial pathway. Exp Toxicol Pathol 63: 69-78, 2011.

43. Zhang H, Fang DC, Lan CH and Luo YH: Helicobacter pylori infection induces apoptosis in gastric cancer cells through the mitochondrial pathway. J Gastroenterol Hepatol 22: 1051-1056, 2007.

44. Yu RX, Hu XM, Xu SQ, Jiang ZJ and Yang W: Effects of fucoxanthin on proliferation and apoptosis in human gastric adenocarcinoma MGC-803 cells via JAK/STAT signal pathway. Eur J Pharmacol 657: 10-19, 2011.

45. Shigeyasu K, Nagasaka T, Mori Y, Yokomichi N, Kawai T, Fuji T, Kimura K, Umeda Y, Kagawa S, Goel A and Fujiwara T: Clinical significance of MLH1 methylation and CpG island methylator phenotype as prognostic markers in patients with gastric cancer. PLoS One 10: e0130409, 2015.

46. Li Y, Yang Y, Lu Y, Herman JG, Brock MV, Zhao P and Guo M: Predictive value of CHFR and MLH1 methylation in human gastric cancer. Gastric Cancer 18: 280-287, 2015.

This work is licensed under a Creative Commons Attribution-NonCommercial-NoDerivatives 4.0 International (CC BY-NC-ND 4.0) License. 